\title{
A new deterministic model of strange stars
}

\author{
Farook Rahaman $^{1, \mathrm{a}}$, Koushik Chakraborty ${ }^{2, \mathrm{~b}}$, P. K. F. Kuhfittig ${ }^{3, \mathrm{c}}$, G. C. Shit ${ }^{1, \mathrm{~d}}$, Mosiur Rahman ${ }^{4, \mathrm{e}}$ \\ ${ }^{1}$ Department of Mathematics, Jadavpur University, Kolkata 700032, West Bengal, India \\ 2 Department of Physics, Government Training College, Hooghly 712103, West Bengal, India \\ ${ }^{3}$ Department of Mathematics, Milwaukee School of Engineering, Milwaukee, WI 53202-3109, USA \\ ${ }^{4}$ Department of Mathematics, Meghnad Saha Institute of Technology, Kolkata 700150, India
}

Received: 13 June 2014 /Accepted: 9 October 2014 / Published online: 30 October 2014

(C) The Author(s) 2014. This article is published with open access at Springerlink.com

\begin{abstract}
The observed evidence for the existence of strange stars and the concomitant observed masses and radii are used to derive an interpolation formula for the mass as a function of the radial coordinate. The resulting general mass function becomes an effective model for a strange star. The analysis is based on the MIT bag model and yields the energy density, as well as the radial and transverse pressures. Using the interpolation function for the mass, it is shown that a mass-radius relation due to Buchdahl is satisfied in our model. We find the surface redshift $(Z)$ corresponding to the compactness of the stars. Finally, from our results, we predict some characteristics of a strange star of radius $9.9 \mathrm{~km}$.
\end{abstract}

\section{Introduction}

The constitution of the interior of neutron stars is still considered an open question by the scientific community. Not only are neutron stars highly dense compact astrophysical objects, the extreme conditions near the center have led to the hypothesis that the core consists entirely of quark matter, in which the constituent quarks comprising the neutrons become deconfined. Moreover, based on the MIT bag model, it was first argued explicitly by Witten [1] that the most stable state of matter is strange quark matter, which is a mixture containing roughly the same number of up, down, and strange quarks.

Quark matter is of interest for a number of reasons. For example, that compact stars could result in a topology change, that is, in the formation of wormholes, had already been suggested in Ref. [2]. It is shown in Ref. [3] that

\footnotetext{
a e-mail: farook_rahaman@yahoo.com

b e-mail: kchakraborty28@yahoo.com

ce-mail:kuhfitti@msoe.edu

de-mail: gopal_iitkgp@yahoo.co.in

e e-mail: rahaman@iucaa.ernet.in
}

the topology change inside a neutron star requires a quarkmatter core of a certain minimal radius. The survival of quark nuggets from the early Universe is also a possibility. Using the MIT bag model, it is shown in Ref. [4] that quark matter may be a suitable candidate for dark matter. In fact, a strange star may be regarded as a huge strangelet.

In $\mathrm{QCD}$, the interaction between quarks becomes weak for a large exchange of momentum. So, for a sufficiently large temperature or density, or both, interaction between constituent quarks becomes very weak and, consequently, they become deconfined. In heavy ion collider experiments deconfinement of quarks may be brought about at high temperatures $(\sim 180 \mathrm{MeV}$ or above). But neutron stars are cold $(\sim$ few $\mathrm{KeV})$. Extremely large chemical potential at the core of the neutron star plays the central role for deconfinement of the quarks. At densities of nearly twice nuclear density, hyperons appear in neutron-star matter and this state is known as the hadronic phase (HP), whereas a deconfined quark matter phase is obtained as a phase transition from the hadronic phase at densities much higher than the nuclear density.

If the conversion of neutron-star matter to quark matter is not confined to the core, then the result is a quark star. Under certain conditions, it is theoretically possible for some up and down quarks to be transformed into strange quarks. Since the strange matter is the true ground state of matter, nothing can stop the conversion of the entire quark star into strange matter once the core gets converted into strange matter. Thus a neutron star gets converted into a strange star. In this paper, we propose a new deterministic model for strange stars based on the MIT bag model.

We organize our paper as follows.

In Sect. 2, we have provided the basic equations. In Sect. 3, we have obtained the solutions of physical parameters. In Sect. 4, we have studied mass-radius relation and surface redshift of the stranger stars. The article is concluded with a short discussion. 


\section{Basic equations}

To describe the spacetime of the interior of the strange star, we assume the metric to be

$d s^{2}=-e^{(r)} d t^{2}+e^{\lambda} d r^{2}+r^{2}\left(d \theta^{2}+\sin ^{2} \theta d \phi^{2}\right)$

and then recall that the most general energy-momentum tensor compatible with spherically symmetry is

$T_{v}^{\mu}=\left(\rho+p_{r}\right) u^{\mu} u_{v}-p_{r} g_{v}^{\mu}+\left(p_{t}-p_{r}\right) \eta^{\mu} \eta_{v}$

with

$u^{\mu} u_{\mu}=-\eta^{\mu} \eta_{\mu}=1$

The Einstein field equations are listed next:

$e^{-\lambda}\left[\frac{\lambda^{\prime}}{r}-\frac{1}{r^{2}}\right]+\frac{1}{r^{2}}=8 \pi \rho$,

Table 1 The values of the mass and radius for various strange stars [57]. The extreme conditions of pressure and density at the center of the neutron stars may lead to a phase transition of the neutrons to hyperons [8], bosons, quark matter or strange quark matter [1]. However, the maximum mass predicted from the consideration of the existence of hyperons or bosons is much smaller than the recently discovered compact objects like J1614-2230 [9] or J0348 +0432 [10]. These obser-

$e^{-\lambda}\left[\frac{1}{r^{2}}+\frac{\nu^{\prime}}{r}\right]-\frac{1}{r^{2}}=8 \pi p_{r}$

$\frac{1}{2} e^{-\lambda}\left[\frac{1}{2}\left(v^{\prime}\right)^{2}+v^{\prime \prime}-\frac{1}{2} \lambda^{\prime} v^{\prime}+\frac{1}{r}\left(v^{\prime}-\lambda^{\prime}\right)\right]=8 \pi p_{t}$.

Our analysis begins with a set of astrophysical objects considered to be candidates for strange stars. The masses and radii of these compact objects are listed in Table 1 . The interpolation technique has been used to estimate the cubic polynomial, which yields the following expression for the mass as a function of the radial coordinate $r$ :

$m(r)=a r^{3}-b r^{2}+c r-d$,

where $a=0.01492, b=0.3296, c=3.03$, and $d=9.6453$. The graph of $m(r)$ is shown in Fig. 1 with residuals corresponding to the observed data and fitted cubic polynomial.

vations practically rule out the possibility of the existence of hyperons, bosons or pure neutrons inside the so called neutron stars. The hadrons inside these stars must get converted to quark or strange quark matter. So, though these compact star candidates are called neutron stars, the theoretical predictions of QCD confirm the transformation of hadrons to quark or strange quark matter. Hence, from theoretical considerations we are safe to consider these compact objects as strange stars

\begin{tabular}{lccc}
\hline Strange stars & Radius $($ in $\mathrm{km})$ & Mass $\left(M_{\odot}\right)$ & The mass in $\mathrm{km}\left(1 M_{\odot}=1.475 \mathrm{~km}\right)$ \\
\hline PSR J1614-2230 & 10.3 & $1.97 \pm 0.04$ & $2.9057 \pm 0.059$ \\
Vela X-1 & 9.99 & $1.77 \pm 0.08$ & $2.6107 \pm 0.118$ \\
PSR J1903+327 & 9.82 & $1.667 \pm 0.021$ & $2.4588 \pm 0.03$ \\
Cen X-3 & 9.51 & $1.49 \pm 0.08$ & $2.1977 \pm 0.118$ \\
SMC X-1 & 9.13 & $1.29 \pm 0.05$ & $1.9027 \pm 0.073$ \\
\hline
\end{tabular}

Fig. 1 Interpolation curves from the observed data of the strange-star candidates with residuals
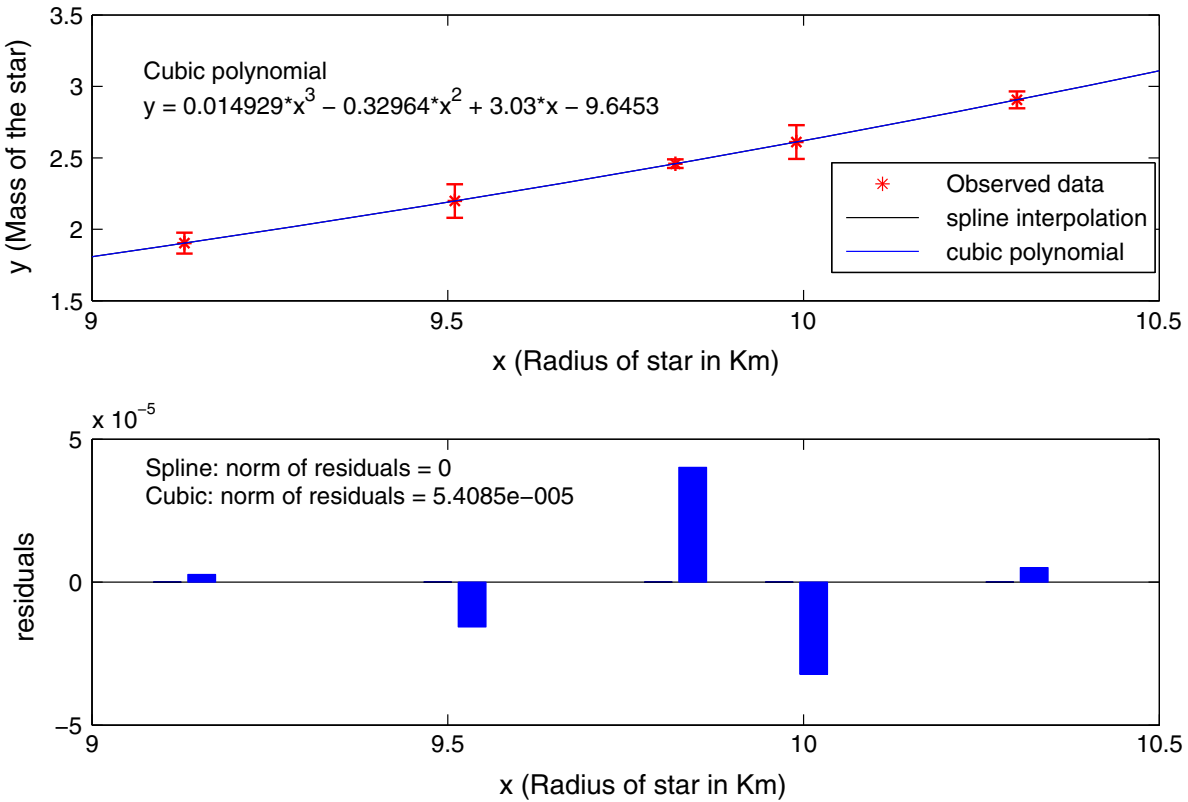


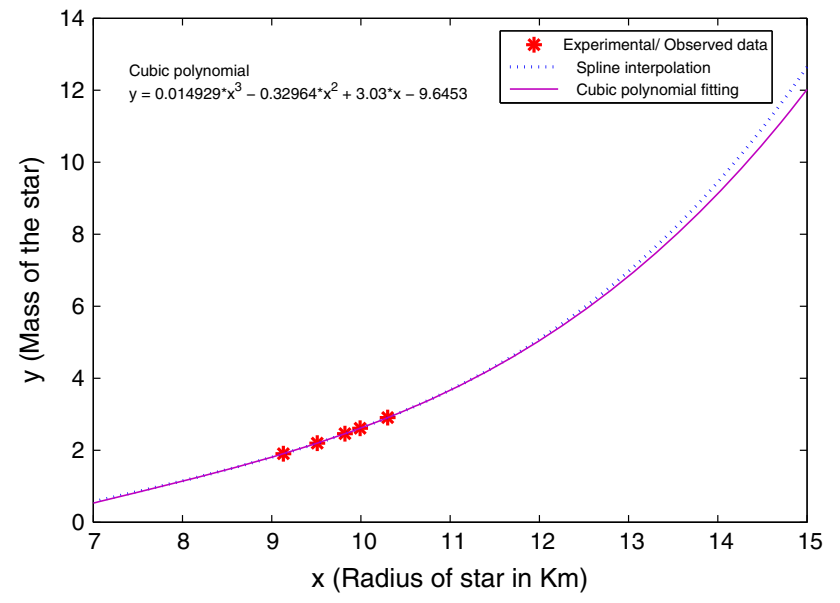

Fig. 2 Interpolation curves from the observed values of mass and radius of the strange-star candidates with extended ranges

From this figure, we notice that the maximum norm of residuals is about $10^{-5}$. The extended range of the mass radius relation is shown in Fig. 2. Observe that the best fit is obtained in the range around $7-12 \mathrm{~km}$. We will see later that the accepted range is $r=6.2 \mathrm{~km}$ to $r=12.2 \mathrm{~km}$. It is generally well known that for any interpolation technique the degree of the polynomial increases with the increase of data points. However, spline interpolation resolves this problem. Because the spline interpolation is a special type of piecewise polynomial so that the interpolation error can be made small even when using low degree polynomials. In our problem, a spline interpolation polynomial is constructed using the given set of observed data for a wide range of radii of the stars. Afterwards we have fitted a cubic polynomial (for mass radius relation) using the data extracted from spline interpolation. Therefore, the use of additional one or more observed data will not significantly affect the results presented here.

Further analysis is going to be based on the MIT bag model. In this model, the strange matter is assumed to have the following equation of state [11]:

$p_{r}=\frac{1}{3}(\rho-4 B)$,

where $B$, the bag constant, is in units of $\mathrm{MeV} /(\mathrm{fm})^{3}$. To obtain a value suitable for our analysis, it is important to note that for the strange-star candidates, $B$ has been found to lie in the range $60-80 \mathrm{MeV} /(\mathrm{fm})^{3}$ for a $\beta$-equilibrium stable strange-matter configuration $[12,13]$. A convenient value for plotting purposes is $B=0.0001$. Since this choice corresponds to $83 \mathrm{MeV} /(\mathrm{fm})^{3}$, it is close to the accepted values. Indeed,

$83 \frac{\mathrm{MeV}}{(\mathrm{fm})^{3}} \frac{G}{c^{4}} \times(10 \mathrm{~m})^{6}=0.0001(\mathrm{~km})^{-2}$.

For other possible values of $B$, see Ref. [14].

\section{Solutions}

From the metric potential $e^{\lambda}$ in Eq. (3), we get

$e^{-\lambda}=1-\frac{2 m(r)}{r}=1-2 a r^{2}+2 b r-2 c+\frac{2 d}{r}$.

Next, making use of Eq. (7), we obtain from Eqs. (3), (4), and (5),

$\rho=\frac{1}{8 \pi}\left(6 a-\frac{4 b}{r}+\frac{2 c}{r^{2}}\right)$,

$p_{r}=\frac{1}{24 \pi}\left(6 a-\frac{4 b}{r}+\frac{2 c}{r^{2}}\right)-\frac{4 B}{3}$,

and

$$
\begin{aligned}
p_{t}= & \frac{1}{16 \pi}\left(1-2 a r^{2}+2 b r-2 c+\frac{2 d}{r}\right) \\
& \times\left(I_{1}-I_{2}+I_{3}+I_{4}-I_{5}\right),
\end{aligned}
$$

where

$$
\begin{aligned}
& I_{1}=\frac{\left(12 a r^{3}-10 b r^{2}-4 c r-\frac{B r^{3}}{2 \pi}-6 d\right)}{2\left(3 r^{2}-6 a r^{4}+6 b r^{3}-6 c r^{2}+6 d r\right)}, \\
& I_{2}=\frac{\left(4 a r-2 b+\frac{2 d}{r}\right)\left(12 a r^{3}-10 b r^{2}-4 c r-\frac{B r^{3}}{2 \pi}-6 d\right)}{2\left(1-2 a r^{2}+2 b r-2 c+\frac{2 d}{r}\right)\left(3 r^{2}-6 a r^{4}+6 b r^{3}-6 c r^{2}+6 d r\right)}, \\
& I_{3}=\frac{\frac{12 a r^{3}-10 b r^{2}-4 c r-\frac{B r^{3}}{2 \pi}-6 d}{3 r^{2}-6 a r^{4}+6 b r^{3}-6 c r^{2}+6 d r}-\frac{4 a r-2 b+\frac{2 d}{r^{2}}}{1-2 a r^{2}+2 b r-2 c+\frac{2 d}{r}}}{r}, \\
& I_{4}=\frac{36 a r^{2}-20 b r-4 c-\frac{3 B r^{2}}{2 \pi}}{3 r^{2}-6 a r^{4}+6 b r^{3}-6 c r^{2}+6 d r}, \\
& I_{5}=\frac{\left(12 a r^{3}-10 b r^{2}-4 c r-\frac{3 B r^{3}}{2 \pi}-6 d\right)\left(6 r-24 a r^{3}+18 b r^{2}-12 c r+6 d\right)}{\left(3 r^{2}-6 a r^{4}+6 b r^{3}-6 c r^{2}+6 d r\right)^{2}} .
\end{aligned}
$$

The plots of Eqs. (9)-(11) are shown in Fig. 3.

\section{Mass-radius relation and surface redshift}

Our final task is to study the maximum allowed mass-toradius ratio in our model. Buchdahl [15] showed that for a perfect-fluid sphere, twice the ratio of the maximum allowed mass to the radius is $8 / 9$, i.e., $2 M / R \leq 8 / 9$. Moreover, if the trace of the energy-momentum tensor is postulated to be nonnegative, then the ratio of the total mass to the coordinate radius is $\leq 5 / 18$. In other words, $M / R$ is strictly less than $4 / 9$. Now, while we would expect the quantity $1-2 M / R$ to be nonnegative, Buchdahl's condition actually does not allow the value to be less than $1 / 9$. 

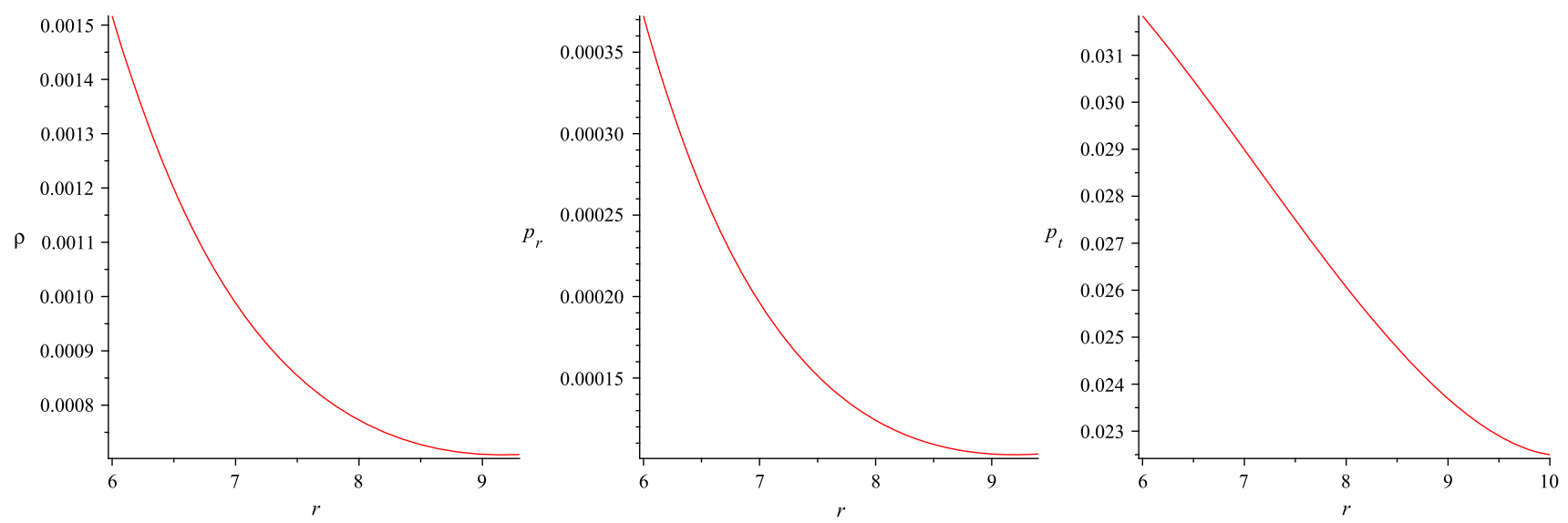

Fig. 3 Left The plot of $\rho$ as a function of $r$ (in km). Middle Plot of $p_{r}$ vs. $r$ (in km) with $B=10^{-4}$. Right Plot of $p_{t}$ vs. $r$ (in km) with $B=10^{-4}$

Table 2 Verification of Buchdahl's condition for various radii

\begin{tabular}{lll}
\hline Radius $(\mathrm{km})$ & $\frac{2 M}{R}$ & Comments \\
\hline 6.1 & -0.013 & Negative \\
6.2 & 0.0086 & $\leq 0.89$ \\
7 & 0.15196 & $\leq 0.89$ \\
8 & 0.28483 & $\leq 0.89$ \\
9 & 0.4008 & $\leq 0.89$ \\
10 & 0.5229 & $\leq 0.89$ \\
11 & 0.6657 & $\leq 0.89$ \\
12.2 & 0.8779 & $\leq 0.89$ \\
12.3 & 0.8999 & $\geq 0.89$ \\
\hline
\end{tabular}

To see how these conditions apply to our model, we return to Eq. (6) and recall that the best fit occurs between the radii 6.2 and $12.2 \mathrm{~km}$. In this range, the condition $1-2 M / R>1 / 9$ is met, as required by Buchdahl's condition. It can be seen from Table 2 that for different radii this condition is obeyed in our model of strange stars.

The compactness of the star is obtained as

$u=\frac{m(r)}{r}=a r^{2}-b r+c-\frac{d}{r}$

The nature of the compactness of the star is shown in Fig. 4 (left). The surface redshift $(Z)$ corresponding to the above compactness $(u)$ is given by

$1+Z=[1-(2 u)]^{-\frac{1}{2}}$,

where

$Z=\frac{1}{\sqrt{2 a r^{2}-2 b r+2 c-\frac{2 d}{r}}}-1$
Table 3 Redshift for various radii

\begin{tabular}{ll}
\hline Radius $(\mathrm{km})$ & $Z$ \\
\hline 7 & 0.0859 \\
8 & 0.1824 \\
9 & 0.29189 \\
10 & 0.4478 \\
11 & 0.7296 \\
11.5 & 0.9924 \\
\hline
\end{tabular}

The redshift $\mathrm{Z}$ can be measured from the X-ray spectrum. This $\mathrm{Z}$ actually gives the compactness of the star. High observed redshifts $(0.35-0.45)$ are consistent with strange stars which have mass-radius ratios higher than neutron stars. Thus, it is easy to find the maximum surface redshift for the anisotropic strange stars of different radii from Eq. 14. We calculate the maximum surface redshift for different strange stars with different radii, which is shown in Table 3. The nature of the surface redshift of the star is shown Fig. 4 (right). Note that since at the surface the radial pressure is zero, i.e. $p_{r}(r=R)=0$, this equation gives a relation between parameters a,b,c,d, and the parameter B. Therefore, the properties of strange matter (comprising the bag constant, B) enter in the redshift value.

\section{Conclusion}

The observed evidence for the existence of strange stars has also led to observed masses and radii. These observations are used in this paper to obtain an interpolation function $m(r)$, which proved to be an effective model for strange stars. The subsequent analysis is based on the MIT bag model and yields both the radial pressure $p_{r}$ and the transverse pressure $p_{t}$, as well as the energy density of the strange star. Subse- 

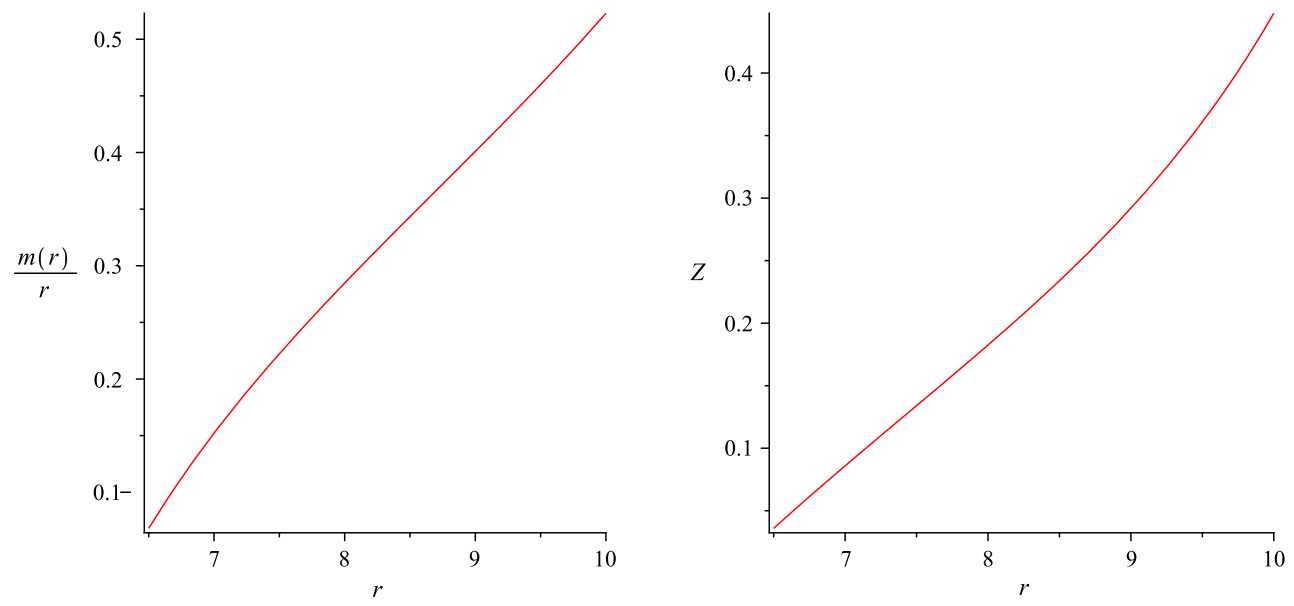

Fig. 4 Left The variation of $\frac{m(r)}{r}$ with respect to $r$ (in $\mathrm{km}$ ). Right The variation of the redshift function with respect to $r$ (in $\mathrm{km}$ )

quently, $m(r)$ was used to show that the mass-radius relation due to Buchdahl is satisfied in our model of a strange star. From our results we can predict some characteristics of a strange star of radius, say $9.9 \mathrm{~km}$. One can see that we have set $c=G=1$ in our calculations. Now, if one substitutes $G$ and $c$ into relevant equations, the value of the surface density of the predicted strange star of radius $9.9 \mathrm{~km}$ turns out to be $\rho_{s}=0.23 \times 10^{15} \mathrm{gm} \mathrm{cm}^{-3}$. Note that our model cannot predict the central density since we cannot get the values of the physical parameters less than $6.2 \mathrm{~km}$ radius. Also, we can predict the mass of strange star of radius $9.9 \mathrm{~km}$ as $1.71 M_{\odot}$. The compactness of the star will be 0.2548 and corresponding redshift is $Z=0.4285$. This high redshift is convenient for explaining strange stars. We can definitely state that our predicted strange star is more compact than neutron stars. In 2008, Cackett et al. [16] reported that redshift of a strange star in the low-mass X-ray binary $4 \mathrm{U} 1820-30$ is $Z=0.43$. This supports our prediction on strange stars in the low-mass X-ray binary 4U 1820-30. Recently, X-ray binaries XTE J1739-285 were suggested as strange stars [17]. We hope our method can be used to determine different characteristics of these strange stars.

Acknowledgments FR and $\mathrm{KC}$ wish to thank the authorities of the Inter-University Centre for Astronomy and Astrophysics, Pune, India, for providing them Visiting Associateship under which a part of this work was carried out. FR is also thankful to UGC, DST for providing financial support. $\mathrm{KC}$ is thankful to University Grants Commission to provide financial assistance under MRP with which this work was carried out We are very grateful to an anonymous referee for his insight- ful comments and constructive suggestions that have led to significant improvements, particularly on the interpretational aspects.

Open Access This article is distributed under the terms of the Creative Commons Attribution License which permits any use, distribution, and reproduction in any medium, provided the original author(s) and the source are credited.

Funded by $\mathrm{SCOAP}^{3}$ / License Version CC BY 4.0.

\section{References}

1. E. Witten, Phys. Rev. D 30, 272 (1984)

2. A. DeBenedictis, R. Garattini, F.S.N. Lobo, Phys. Rev. D 78, 104003 (2008)

3. P.K.F. Kuhfittig, Adv. Math. Phys. 2013, 630196 (2013)

4. F. Rahaman, P.K.F. Kuhfittig, R. Amin, G. Mandal, S. Ray, N. Islam, Phys. Lett. B 714, 131 (2012)

5. P.B. Demorest et al., Nature 467, 1081 (2010)

6. P.C.C. Freire et al., MNRAS 412, 2763 (2011)

7. M.L. Rawls et al., ApJ 730, 25 (2011)

8. A.W.G. Cameron, ApJ. 130, 884 (1959)

9. P. Demorest et al., Nature 467, 1081 (2010)

10. J. Antoniadis et al., Science 340, 6131 (2013)

11. F. Rahaman, R. Sharma, S. Ray, R. Maulick, I. Karar, Eur. Phys. J. C 72, 2071 (2012)

12. E. Farhi, R.L. Jaffe, Phys. Rev. D 30, 2379 (1984)

13. C. Alcock, E. Farhi, A. Olinto, Astrophys. J. 310, 261 (1986)

14. M. Kalam, A.A. Usmani, F. Rahaman, S.M. Hossein, I. Karar, R. Sharma, Int. J. Theor. Phys. 52, 3319 (2013)

15. H.A. Buchdahl, Phys. Rev. D 116, 1027 (1959)

16. E.M. Cackett et al., ApJ 674, 415 (2008)

17. T.J. Maccarone, R. Bandyopadhyay, J. Kennea, The Astronomer's Telegram, 4354 Publication Date: 09/2012 